McElroy, T., \& Conrad, J. (2009). Thinking about product attributes: Investigating the role of unconscious valence processing in attribute framing. Asian Journal of Social Psychology, 12, 157-161. Published by Wiley-Blackwell (ISSN: 1367-2223). DOI: 10.1111/j.1467-839X.2009.01281.x The definitive version is available at www3.interscience.wiley.com

\title{
Thinking about product attributes: Investigating the role of unconscious valence processing in attribute framing
}

Todd McElroy and Jacob Conrad

\begin{abstract}
In the present investigation we conducted three studies to examine how unconscious valence processing influences participants' quality judgments in an attribute-framing task. In Studies 1 and 2 we observed how individuals who had depleted cognitive resources, through distraction (Study 2) and time constraint (Study 3), differed in their responses to an attribute-framing task. In Study 3 we subliminally primed participants with attribute frames and then presented them with a frameless decision task. Our results revealed that attribute framing arises from unconscious valence processing and conscious processing may only play a role when the frame is especially salient.
\end{abstract}




\section{INTRODUCTION}

Every day we face numerous situations that require us to evaluate and decide among alternatives. The processes involved in how we evaluate and make choices have garnered considerable research attention. At the heart of this interest lies the belief that a better understanding of the processes involved in how people evaluate alternatives and make decisions should allow for more accurate and unbiased decision-making.

Inevitably, when we are faced with decisions, select words are used for presentation. Most research has dealt with decision tasks that present participants with alternatives of positive or negative valences, commonly referred to as the frame (Kahneman \& Tversky, 1979). Research has shown that how decision problems are framed has a profound influence on the alternatives that people choose (see Kühberger, 1998; Levin, Schneider \& Gaeth, 1998 for reviews). Prior research (Levin et al., 1998) has identified three fundamentally different ways or 'typologies' that researchers have used to manipulate the frame: riskychoice, goal and attribute framing. Most research investigating framing effects has focused on risky-choice framing. The current study is designed to focus on the processes involved in attribute framing, an area that has not been widely investigated. Attribute framing involves manipulating the wording of objectively equivalent information. The task is designed so that the framing manipulations will either accentuate the positive aspects of the target, or accentuate the negative aspects. For example, Levin and Gaeth (1988) asked participants to evaluate ground beef that was labelled as either $75 \%$ lean or $25 \%$ fat. In their study, they found that the ground beef was rated more positively when it was labeled as $75 \%$ lean as opposed to $25 \%$ fat. While the parameters of attribute framing were clearly defined by Levin et al. (1998), research has not examined the processes that may underlie this effect.

\section{Subliminal priming}

When a stimulus prime is presented subliminally, it can have profound influences on later judgments without the person's conscious awareness. Further, activation of valence information (positive or negative) seems to precede other forms of information (e.g. Bargh, Chaiken, Govender \& Pratto, 1992). Therefore, without the person being consciously aware, subliminally presented stimuli can influence valence reactions toward a stimulus (e.g. Chen \& Bargh, 1999; McConnell \& Leibold, 2001). By automatically 
activating either positive or negative valences, the primed stimulus exerts its valence on an otherwise ambiguous target (e.g. Stapel \& Koomen, 2005). Therefore, if the positive or negative valence of a task carries weight in the decision-making process, then one could predict that priming valence words, either positive or negative, will influence how decision-makers evaluate ambiguous targets. That is to say, if a positively valenced prime is subliminally presented, it should automatically activate positive-related information and exert its influence on the task at hand; negatively valenced primes should function in a similar manner.

Further evidence for the influence of automatic valance information can be found in research examining the selective accessibility model (Mussweiler, 2003). According to this model, knowledge is made accessible through positive or negative social comparisons and, in turn, influences individuals' self-evaluations. In a more relevant extension of the model, Mussweiler \& Englich (2005) demonstrated that participants assimilate their judgments of a target toward subliminally presented stimuli (i.e. the anchor). The finding that individuals gravitate their decisions toward subliminally presented information supports our assertion that attribute-framing effects are largely unconscious.

\section{Current investigation}

In the current set of studies we attempted to investigate the role of automatic valence processing in attribute framing. Specifically, we wanted to examine whether attributeframing effects, similar to other valence-based behaviours (e.g. Chen \& Bargh, 1999), are rendered by automatic processes, or whether they involve more conscious processing. If very early valence priming is found to influence decision choice, then this suggests a decision-making process that is predicated by automatic-evaluative tendencies that act to shape later decision choice.

To examine this question, we conducted a series of studies designed to test the role that unconscious processing plays in attribute-framing effects. In studies 1 and 2 we relied on procedures designed to diminish conscious resources and allow for relatively more influence of unconscious processing. If attribute framing relies more upon conscious processing, then framing effects should be attenuated when conscious resources are diminished. Alternatively, if attribute framing effects are largely due to unconscious processing, then there should be little or no difference in the strength of the framing effect when 
cognitive resources are taxed. In study 3 , we wanted to further examine our hypothesis using methodology that is free of cognitive load. To do so, we subliminally primed participants with either a positive or a negative attribute frame and presented them with a 'frameless' attribute decision task. Consistent with prior research, we hypothesized that for Studies 1 and 2 placing participants under cognitive load would not substantially reduce attribute framing. In Study 3 we hypothesized that when the valence frame is primed subliminally, the unconsciously activated frame will exert its influence and colour the subsequent evaluation of a 'valence neutral' framing task.

\section{EXPERIMENT 1}

\section{Method}

Participants and design. Eighty participants took part in this investigation. The design of our study was a 2 (distraction, no distraction) $\times 2$ (frame: positive, negative frame) factorial design. Participants' quality ratings of the target object (beef) acted as our dependent variable.

Procedure. After arriving for the study, participants were assigned to a condition of distraction or no distraction. Participants in the distraction condition were seated at a computer and given headphones. After getting comfortable and adjusting their headphones, they were given written instructions that informed them they would be answering a series of questions, while listening to a 5 min audio file that contained a short story. Additional instructions required subjects to count the number of times they heard the word 'green' within the short story.An audio file was prearranged in their computer terminal. After the audio file had begun, participants were instructed to turn over the paper in front of them and begin answering the questions. Participants were given a questionnaire that contained the beef attribution task and asked to rate the quality of the beef on a seven-point scale ranging from 'low quality' to 'high quality'. This was followed by three subsequent questions used to measure the effect of framing. After listening to the audio file, subjects indicated the total number of times they heard the word 'green' within the short story.

Participants in the no-distraction condition were seated in the same computer terminal and allowed to get comfortable. They were then provided with written instructions informing them that they would be answering a series of questions. Participants were then instructed to turn over the paper and begin. The stimuli contained the attributeframing task and a series of questions. After completing the 
task, all participants were debriefed and thanked for their participation.

\section{Results}

In accordance with our hypothesis, if attribute-framing effects are largely a function of automatic processing, then the distraction manipulation should not influence the attribute-framing effect. Alternatively, if conscious processing does play a significant role in attribute framing, then distracting a person should lessen the framing effect. To test this prediction, we carried out a $2 ¥ 2$ anova with level of distraction and frame acting as the independent variables. Participant's quality rating of the target (beef) acted as the dependent variable.1 The results of this analysis revealed a main effect for the attribute-frame condition $F(1,76)=$ 21.83, $p<0.001$. However, the interaction between attribute frame and level of distraction did not approach significance $F(1,76)=0.12, p>0.7$ and neither the positive nor the negative framing condition appeared to render any effects from the distraction (Table 1).

Table 1 Average quality rating as a function of level of distraction and attribute frame

\begin{tabular}{lll}
\hline & $N$ & Average \\
\hline Cognitive distraction & & \\
Frame: & & \\
$\quad$ Positive (lean) & 20 & 5.8 \\
$\quad$ Negative (fat) & 20 & 4.85 \\
No distraction & & \\
Frame: & & \\
$\quad$ Positive (lean) & 20 & 5.8 \\
$\quad$ Negative (fat) & 20 & 4.7 \\
\hline
\end{tabular}

\section{EXPERIMENT 2}

In Study 1 we used a distraction task to test whether depleting cognitive resources would influence the strength of attribute framing. This methodology represents a traditional means for testing such an assumption and the null finding supports our hypothesis. However, in Study 2 we wanted to use a different type of methodology and also alter the presentation of the scenario. Therefore, in Study 2 we used a time-pressure technique to manipulate conscious resources and again tested whether conscious processing 
appears to play a substantial role in attribute framing. Further, we also manipulated the percentage information for the target to maximize the conditions for observing conscious involvement.

\section{Method}

Participants and design. One-hundred and twenty undergraduate students at Appalachian State University took part in the study. Participants were randomly assigned to the time-pressure or no-pressure condition as well as the positive, negative attribute-framing conditions. This yielded a 2 (time pressure, no restriction) $\times 2$ (positive frame, negative frame) between-subjects factorial design. The dependent variable in this study was the quality rating assessment question used in Study 1.

Table 2 Average quality rating as a function of time constraint and attribute frame

\begin{tabular}{llc}
\hline & $N$ & Average \\
\hline Time pressure & & \\
$\quad$ Frame: & & \\
$\quad$ Positive (lean) & 30 & 4.3 \\
$\quad$ Negative (fat) & 30 & 3.5 \\
No time pressure & & \\
$\quad$ Frame: & & \\
$\quad$ Positive (lean) & 30 & 4.3 \\
$\quad$ Negative (fat) & 30 & 2.8 \\
\hline
\end{tabular}

Procedure and materials. After agreeing to take part in the study, participants were given one of two sets of instructions prior to beginning the attribute-framing task. Participants in the no time-pressure condition were not given time restriction and were told to begin the task and turn the paper over when they finished. Participants in the time-pressure condition were told that they had $20 \mathrm{~s}$ to complete the task. Prior testing had showed this to be the optimal amount of time for participants to actually complete the task but still feel very hurried in doing so. The experimenter was visible in front of each participant and noted the time on a time-keeping device. After reading the instructions, participants were told to turn the paper over and begin; at this interval the experimenter began timing the task. After $20 \mathrm{~s}$, participants were instructed to stop working and turn the paper over. The task used in this study was the same as that used in 
Experiment 1 except that in this study we altered the percentages of fat and lean to $50 \%$ in both framing conditions.

Specifically, in Study 1, the beef was represented as it has been traditionally, $75 \%$ lean and $25 \%$ fat. We reasoned that, by modifying the probabilities to an even $50 \%$, this should reduce the framing effect in both conditions if individuals consciously processed the percentage from an expected value approach, or it may act to maximize saliency to participants, so that conscious processing would be observed [2].

\section{Results}

Similar to Study 2, we wanted to explore whether depletion of cognitive resources would influence the strength of attribute framing. To explore this question we again performed a $2 \times 2$ anova with level of cognitive capability (time pressure, no time pressure) and attribute frame (positive, negative) serving as our independent variables. Participants' quality rating of the target (beef) acted as our dependent variable. The results from this analysis revealed a significant main effect for attribute framing $F(1,116)=28.5$, $p<0.001$. As predicted, the interaction between time pressure and framing did not reach a level of statistical significance $F(1,116)=2.6, p<0.11$. As can be seen in Table 2, the means for the positive-framing condition are equal, and the effect is largely driven by the negative-framing condition. To investigate whether individuals in the losses condition may have been significantly affected by our time-pressure manipulation, we performed a post-hoc analysis, focusing solely on the negative framing ' $50 \%$ fat' condition. This analysis revealed that individuals who were not cognitively constrained by time pressure rated the quality of the beef significantly lower than those individuals who were under time pressure $F(1,58)=4.04, p<0.05$.

\section{EXPERIMENT 3}

In Studies 1 and 2 we used methodology that limited the availability of cognitive resources. In doing so, we found that attribute-framing effects still occur, suggesting this effect is due to unconscious processing. In our third study we used subliminal priming, a method that tests unconscious processing without depleting cognitive resources.

\section{Method}

Participants and design. The participants in our study were 100 Appalachian State University undergraduate students. 
All of our participants received class credit for taking part in the study. The design of our study was a one-way factorial design with valence of primed stimulus (negative, positive) acting as our independent variable, and participant's evaluation of the decision task as our dependent variable.

Materials and procedure. After consenting to take part in the study, participants were seated at a computer and told that they would be asked for their opinion about a task. They were then asked to follow the instructions presented to them on the monitor. Participants were further told that during the study a fixation symbol ' + ' would appear in the centre of the screen and they should focus their attention at that point. After pressing the space bar, participants were presented with the task introduction used in Studies 1 and 2. Specifically, participants were asked to: 'Assume that you are inviting a special friend to dinner and that you are making lasagna with ground beef'. Next, they were presented with the attribute frame (i.e. 'fat' or 'lean') subliminally. The attribute frame was flashed in the centre of the screen for $30 \mathrm{~ms}$. The prime was followed directly afterward

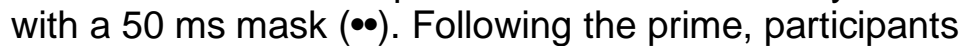
were asked to evaluate the beef on the same scale used in the previous studies. After completion of the task, all of our participants were provided with a funnel debriefing measure wherein they were asked questions building toward ascertaining knowledge of the subliminal prime (Bargh \& Chartrand, 2000). Afterward, participants completed an unrelated paper and pencil task and were debriefed from the study.

\section{Results}

To investigate our hypothesis we carried out a one-way anova3 with subliminal prime valence (positive, negative) acting as our independent variable, and participants' evaluation scores serving as our dependent variable. The results of this analysis revealed a significant effect for valence of subliminal prime $F(1,92)=6.97, p<0.01$. Consistent with prior research, when the positive frame (lean) was subliminally presented, it generated higher quality ratings $(M=5.9)$ than the subliminally presented negative frame (fat) $(M=5)$. These data indicate that the subliminal presentation of valence primes profoundly influenced participant's evaluation. 


\section{DISCUSSION}

In our investigation we examined the unconscious nature of attribute framing across three studies. In the first two studies we discovered that when cognitive resources are limited (i.e. distraction and time constraint) attribute framing effects still occur, providing good evidence that this effect is carried out by unconscious processing. In our third study, we subliminally primed the attribute frames and observed their effects on participants' choices. This experiment demonstrated that unconscious priming of valence information can have wieldy and predictable effects on a valence neutral attribute task. This finding was consistent with the previous two studies and supported our hypothesis that attribute-framing effects are largely a product of unconscious processing.

While our studies provide strong support for the influence of unconscious processing in attribute framing, the findings in the losses condition of Study 3 may seem somewhat surprising and intriguing, specifically, the finding that participant's quality ratings were lower for the negative target (fat) when there was no time pressure. Although our reasoning is post-hoc, we believe that the negativity from the $50 \%$ fat beef was so profound that it caused participants to become 'attentive' to this undesirable information. Consequently, when participants had time to consciously process this information they rated the $50 \%$ fat beef as lower in quality than participants who were under time pressure and had no opportunity to consciously evaluate this information. In essence, modifying the percentages appeared to magnify the valence of the negative attribute frame and induce conscious processing. This observation seems well grounded, even pronounced by Kahneman and Tversky's (1979) classic depiction that 'losses loom larger than gains'.

Our findings also open the door to future research investigating how automatic evaluations influence subsequent decision-making. For example, there has been a good deal of recent interest in how the style of processing (McElroy \& Seta, 2003; Igou \& Bless, 2007; Stanovich, 2008), memory retrieval (Reyna \& Brainerd, 1991), emotional-rational inconsistencies (Wang, 2006) and individual differences (Chatterjee, Heath, Milberg \& France, 2000; Levin, Gaeth, Scheriber \& Lauriola, 2002; Xie \& Wang, 2003; Simon, Fagley \& Halleran, 2004; Wang, 2004; McElroy \& Seta, 2007; McElroy, Seta \&Waring, 2007; Dickinson \& Drummond, 2008) play out in the framing process and decision choice. Further, this may provide insight on recent findings that show incompatibility between trust and choice 
in decision-making (Keren, 2007). While these factors have all yielded insight into how the framing process occurs, future research should examine how automatic and unconscious processing operates in conjunction with these processes to better understand the decision-making process.

\section{NOTES}

1. All of the remaining three questions relevant to the target were highly correlated with each other as well as question 1 . We wanted to focus our analysis on question 1 to maintain consistency and comparability across the studies. Further, when we used the average across the items, all of the statistical results remained consistent with the reported data on question 1.

2. Special thanks to Yoshi Kashima for directing us to this question.

3. While none of the participants guessed the hypothesis of our study, six of the participants indicated that they saw words similar in nature (and valence) to our subliminal primes; therefore, we excluded them from further analyses.

\section{REFERENCES}

Bargh, J. A., Chaiken, S., Govender, R. \& Pratto, F. (1992). The generality of the automatic evaluation activation effect. Journal of Personality and Social Psychology, 62, 893-912.

Bargh, J. A. \& Chartrand, T. L. (2000). The mind in the middle: A practical guide to priming and automaticity research. In: H. Reis \& C. Judd, eds. Research Methods in Social Psychology, pp. 253-285. New York: Cambridge University Press.

Chatterjee, S., Heath, T. B., Milberg, S. J. \& France, K. R. (2000). The differential processing of price in gains and losses: The effects of frame and need for cognition. Journal of Behavioral Decision Making, 13, 61-75.

Chen, M. \& Bargh, J. A. (1999). Consequences of automatic evaluation: Immediate behavioral predispositions to approach and avoid the stimulus. Personality and Social Psychology Bulletin, 25, 215-224.

Dickinson, D. L. \& Drummond, S. P. A. (2008). The effects of total sleep deprivation on Bayesian updating. Judgment and Decision Making, 3, 181-190. 
Igou, E. R. \& Bless, H. (2007). On undesirable consequences of thinking: Framing effects as a function of substantive processing. Journal of Behavioral Decision Making, 20, 125142.

Kahneman, D. \& Tversky, A. (1979). Prospect theory:An analysis of decision under risk. Econometrica, 47, 263-291.

Keren, G. (2007). Framing, intentions, and trust-choice incompatibility. Organizational Behavior and Human Decision Processes, 103, 238-255.

Kühberger, A. (1998). The influence of framing on risky decisions: A meta-analysis. Organizational Behavior and Human Decision Processes, 57, 23-55.

Levin, I. P., Gaeth, G. J. (1988). How consumers are affected by the framing of attribute information before and after consuming the product. Journal of Consumer Research, 15, 374-378.

Levin, I. P., Gaeth, G. J., Schreiber, J. \& Lauriola, M. (2002). A new look at framing effects: Distribution of effect sizes, individual differences, and independence of types of framing. Organizational Behavior and Human Decision Processes, 88, 411-429.

Levin, I. P., Schneider, S. L. \& Gaeth, G. J. (1998). All frames are not created equal: A typology and critical analysis of framing effects. Organizational Behavior \& Human Decision Processes, 76, 149-188.

McConnell, A. R. \& Leibold, J. M. (2001). Relations among the implicit association test, discriminatory behavior, and explicit measures of racial evaluations. Journal of Experimental Social Psychology, 37, 435-442.

McElroy, T. \& Seta, J. J. (2003). Framing effects: An analyticholistic perspective. Journal of Experimental Social Psychology, 39, 610-617.

McElroy, T. \& Seta, J. J. (2007). Framing the frame: How task goals determine the likelihood and direction of framing effects. Judgment and Decision Making, 2, 251-256.

McElroy, T., Seta, J. J. \& Waring, D. (2007). Reflections of the self: How self-esteem determines decision framing and increases risk taking. Journal of Behavioral Decision Making, 20, 223-240. 
Mussweiler, T. (2003). Comparison processes in social judgment:

Mechanisms and consequences. Psychological Review, 110,

472-489.

Mussweiler, T. \& Englich, B. (2005). Subliminal anchoring: Judgmental consequences and underlying mechanisms. Organizational

Behavior and Human Decision Processes, 98, 133-143.

Reyna, V. F. \& Brainerd, C. J. (1991). Fuzzy-trace theory and framing effects in choice: Gist extraction, truncation, and conversion.

Journal of Behavioral Decision Making, 4, 249-262.

Simon, A. F., Fagley, N. S. \& Halleran, J. G. (2004). Decision

framing: Moderating effects of individual differences and

cognitive processing. Journal of Behavioral Decision Making,

17, 77-93.

Stanovich, K. E. (2008). Higher-order preferences and the Master

Rationality Motive. Thinking \& Reasoning, 14, 111-127.

Stapel, D. A. \& Koomen, W. (2005). Multiple ambiguity and an impression formation focus make inapplicable prices useful.

Social Cognition, 23, 324-335.

Wang, X. T. (2004). Self-framing of risky choice. Journal of Behavioural Decision Making, 17, 1-16.

Wang, X. T. (2006). Emotions within reason: Resolving conflicts in risk preference. Cognition and Emotion, 20, 1132-1152.

Xie, X.-F. \& Wang, X. T. (2003). Risk perception and risky choice: Situational, informational and dispositional effects. Asian Journal of Social Psychology, 6, 117-132. 